\title{
Tooltip Translator for Social Networking
}

\author{
L. B. Krithika ${ }^{1 *}$, Anand Mahendran ${ }^{2}$, A. Kalyana Vasanth ${ }^{1}$, G. R. Reddy Harish ${ }^{1}$ and T. Hariharan ${ }^{1}$ \\ 'School of Information and Technology and Engineering \\ ${ }^{2}$ School of Computing Science and Engineering, VIT University, Vellore, Tamilnadu, India; \\ krithika.lb@vit.ac.in
}

\begin{abstract}
Natural language processing (NLP) has become a main context for many websites. When we consider the case of social networking websites, many posts and conversations in the present day lacks in punctuation and vocabulary. People use short forms such as wru (where are you), hru (how are you) etc., in messages and comments in social networking sites. The comments with respect to the posts need to be categorized into positive, neutral or negative comments. Suppose a person post a status and people start commenting on it, there should be a measure above the post indicating the percentage of positive, negative and neutral comments. Hence, there is need for developing a tool-tip translator, which expands such short forms when mouse cursor is placed on them. There is also a need for developing a text classification system that classifies text based on polarity. Positive comments will be marked in green colour and negative comments will be marked in red colour and neutral in yellow. This paper shows how to implement such a tooltip translator and text classification system.
\end{abstract}

Keywords: Natural Language Processing, Opinion Mining, Tooltip Translator, Sentiment Analysis, Social Networking

\section{Introduction}

Opinion mining determines a user or speaker's view on some topic in terms of polarity. Polarity for example can be expressed as positive, negative or neutral. For a better understanding, we will consider a social networking site. Let us assume that a user has commented on a post or a photo. Opinion mining determines the polarity of this comment. That is, whether the comment is positive, negative or neutral with respect to the photo or the post. For example: "the photo looks dull" indicates a negative comment.

Given a set of texts, our objective is to extract the features and determine the opinion. That is, whether the text is positive, negative or neutral. News can be positive, negative or neutral. Using sentiment analysis techniques such as chunking and rule based grammar, we can determine how the news can impact important entities such as politics ${ }^{1}$. Before the internet was popular, whenever a person wants to make a decision making, he/she had to consult his/her parents, relatives and friends. And also if an organization wants to know about the quality of its product, it has to make a survey ${ }^{2}$. So, there has to be a way in which the opinions are readily available for people to make decisions and hence it was identified that there is a need for Opinion mining.

Consider an e-commerce website like flip kart, where the site allow users to post their reviews about the products purchased. These reviews are called "user generated content". For example, user writes a review on the book that he has purchased and gives a rating to the book out of 5 . Now people no longer need to consult family or friends if they want to buy a product. They just need to go through these reviews that give them an insight about the product that they are going to purchase.

Opinions are extracted from the reviews given by the users and these reviews are hence classified as positive, negative or neutral. This helps users to find opinion about the product for which they are searching.

${ }^{*}$ Author for correspondence 


\section{Related Work}

Social networking sites are used by almost every computer user of the present day. These sites allow users to share their thoughts and updates. Some of the popular social networking sites of the present day are facebook, twitter, googleplus and myspace. Sentiment analysis on twitter has gained notice because of increasing number of registered users and enormous number of tweets every hour. Many methods have been proposed to classify tweets and to determine the polarity of the messages. But due to the slang and spelling mistakes, it becomes difficult to determine the exact polarity ${ }^{3}$. The existing tools for POS tagging, chunking and named entity recognition when applied on tweets perform in a poor manner. Due to their brief nature, tweets frequently lack sufficient context to recognize the types of the entities they contain ${ }^{4}$.

\subsection{Efforts Made in Classifying the Tweets}

One of the significant efforts for sentiment classification was opinion extraction from biased and noisy data. This method predicted polarity from three popular websites to train a model. They use thousand manually labelled tweets for training and another thousand manually labelled tweets for testing. They did not reveal how they collect this test data ${ }^{5}$. As most of the tweets in twitter are public, it is possible to mine them. Immediately classifying the tweet as positive, negative or neutral brings a challenge.

Weka data mining API was used to classify the tweets. This API uses machine learning approach for classifying the tweets in Java programming language $e^{6}$. As it is important for a company to know the opinion of the customers on their products, it is necessary to classify the tweets related to that particular company's products. Opinion mining over twitter space can be done effectively using $\mathrm{R}$ programming language ${ }^{7}$. R programming language is used for statistical computing and graphics. It was shown that usage of $\mathrm{R}$ programming language reduces task of opinion mining and sentiment analysis ${ }^{7}$. A method to determine the social interest of people in regard to a social event was developed. Opinion of people about specific political candidates with regard to Australian federal election 2010 event was shown. This type of opinion was extracted using Tweets Sentiment Analysis Model (TSAM) which analyses "Web 2.0" user generated content ${ }^{8}$. In the next section we will discuss about Tooltip translator.

In social networking sites the ways of expression and conversation have changed rapidly over the years. With the increase in number of users, the number words that are used to frame a sentence is decreased and almost is being confined to a fixed number of characters (ex: twitter feed, Short Message Service). This tends the user to go for short form of the words which are technically called as "noisy words". Not everyone is familiar with these words as individual choice of short forms and usage of short forms differs and addictiveness to social networking sites varies. So, there is a need to make even the naïve users aware of it with ease.

A survey has been made on Chinese students to know the means by which they find the meaning of unknown words. Survey showed that majority of them used a dictionary like pocket dictionary, digital dictionary or a dictionary in phone. So the need of a tooltip translator was recognized for them 9 . We have extended the same idea to the domain of social networking in this paper. When a user encounters such kind of short forms, they have to look into the websites which offer expansions of the short forms. The short forms like omg(oh my god), hru(how are you)are also called as internet slangs coined in the social networking sites.

With respect to natural language processing, there are lot of toolkits available and research that is being done for automatically extracting and organizing acronyms and expansions (example- WRU for Where Are You) in text ${ }^{10}$.Several approaches to extract acronym/expansion pairs from text have been proposed. These include rule/ pattern based approaches ${ }^{11-17}$ and machine learning based approaches ${ }^{18}$. So a toolkit to deal with, such kind of short forms of words in social networking domain is very new to the field of natural language processing.

\section{Proposed System}

Tooltip translator and sentiment analysis of social networking texts, can be implemented with accuracy using PHP, JavaScript and MYSQL. Though many natural language processing toolkits available like NLTK and Stanford NLP, this method of extracting sentiment from text using PHP, MYSQL and JavaScript is new and easier.

In this section, four text documents for storing positive unigrams, negative unigrams, positive bigrams and negative bigrams are created. Unigrams are single words. Bigrams are combination of two words. A function has been designed to obtain the polarity of a message, on passing the message as a parameter into the function. When a message is passed as a parameter to this function, 
the function splits this message into individual words. Each individual word is checked for a match in positive unigram and negative unigram text files. After that, the sentence is split into chunks of two words (to match bigrams) each. Now these chunks are compared with positive bigrams and negative bigrams text files. Polarity is determined based on the match found. The function returns a value on a scale of 0 to 100 . This value is determined by,

$$
\text { Polarity }=[P /(P+N)]^{*} 100
$$

where,

$$
\begin{aligned}
& \mathrm{P}=\sum(\mathrm{Ui}+\mathrm{Bi}) \\
& \mathrm{N}=\sum(\mathrm{Uj}+\mathrm{Bj})
\end{aligned}
$$

where, $1<=\mathrm{i}<=\mathrm{n}$

and $1<=\mathrm{j}<=\mathrm{n}$

where $\mathrm{n}$ is the total number of unigrams or bigrams respectively. And unigram is given as $\mathrm{U}$ and bigram is given as $B$.

Now the text is classified as positive, negative or neutral based on the polarity value. If the polarity is greater than 50 , it is classified as a positive text. Else if, the polarity is less than 50 , it is classified as a negative text. If the polarity is equal to 50 , it is classified as a neutral text.

We have created a MYSQL table with two columns, one for short forms or compressed text and the other for full form or actual text. We populated this table with popular social networking compressed texts (such as gr8 is the compressed text for great). We created a function that takes a word as a parameter and returns its actual form or full form after performing a search in the MYSQL table. This full form can be displayed on hover using HTML acronym tag.

\section{Results and Discussion}

The system has been totally implemented using PHP, MYSQL and JavaScript. The implementation makes it easier to classify social texts than any other natural language processing toolkit. There are three different modules to be discussed namely Sentiment module, Tooltip module, Trends module.

\subsection{Sentiment Module}

Four text files with names "positive_unigram.txt", "negative_unigram.txt", "positive_bigram.txt" and "negative_bigram.txt" have been created. These text files have been populated with positive unigrams, negative unigrams, positive bigrams and negative bigrams respectively. Variables with names "pos", "neg", "polarity" have been created and were initialized to ' 0 '. We then create a PHP function with name "sentiment" which takes a sentence or a paragraph as a parameter. We split the sentence into single words and chunks of two words using the PHP "explode" function. We then compare each single word with the words in "positive_unigram.txt" and "negative unigram.txt". If a match is found in "positive_unigram. txt", we increment pos by 1 . If a match is found in "negative_unigram.txt", we increment neg by 1. Likewise each chunk of two words is compared with the words in "positive_bigram.txt" and "negative_bigram.txt". And pos and neg are incremented by 1 , depending on whether the match is found in "positive_bigram.txt" or "negative_ bigram.txt" respectively. The polarity is determined by the following equation.

$$
\text { Polarity }=[\text { pos } /(\text { pos }+ \text { neg })] 100
$$

The value of the polarity is retrieved into a HTML "span" tag. We create a "span" tag at the end of every message. This span tag holds the value of polarity. For Example: $<$ span $>80</$ span $>$. To change the colour of the text, we retrieve the polarity of individual messages in a page by using "getElementsByTagName" function in JavaScript whose parameter is "span" (getElementsByTagName ('span')). We then analyze the polarity present in the span tag. If the polarity is greater than 50 , we set the text colour to be green. If, the polarity is less than 50 , we set the text colour to be red. Finally if the polarity is equal to 50, we set the text colour to be yellow. We set the colour of text using the "setAttribute" function in JavaScript.

The following is the algorithm for Sentiment module,

function sentiment(String text)
begin
var sentiment_positive, sentiment_negative
Split "text" over space
var unigram_positive, unigram_negative
load positive_unigram.txt into unigram_positive
load negative_unigram.txt into unigram_negative
for var $i=0$ until i<number of words in text
begin

if(pattern match is found between a word in text and a word in unigram_positive)

then

increment sentiment_positive by 1 
if(pattern match is found between a word in text and a word in unigram_negative)

then var total_sentiment increment sentiment_negative by 1

total_sentiment $=[$ sentiment_positive/(sentiment_ positive + sentiment_negative) $]^{\star} 100$

if(total_sentiment $>50)$

then

print text in green colour

if(total_sentiment< $<0)$

then

print text in red colour

if(total_sentiment $=50)$

then

end

print text in yellow colour

end

The Figure 1 shows messages page in the website. Messages are displayed with the timestamp on which they were posted. The message appears in red, green or yellow depending on the polarity as negative, positive and neutral respectively.

\subsection{Tooltip Module}

The words in a message are fragmented using the PHP "explode" function and are passed into a function with name "extract_acro". In the "extract_acro" function, we match the word that have been passed as a parameter

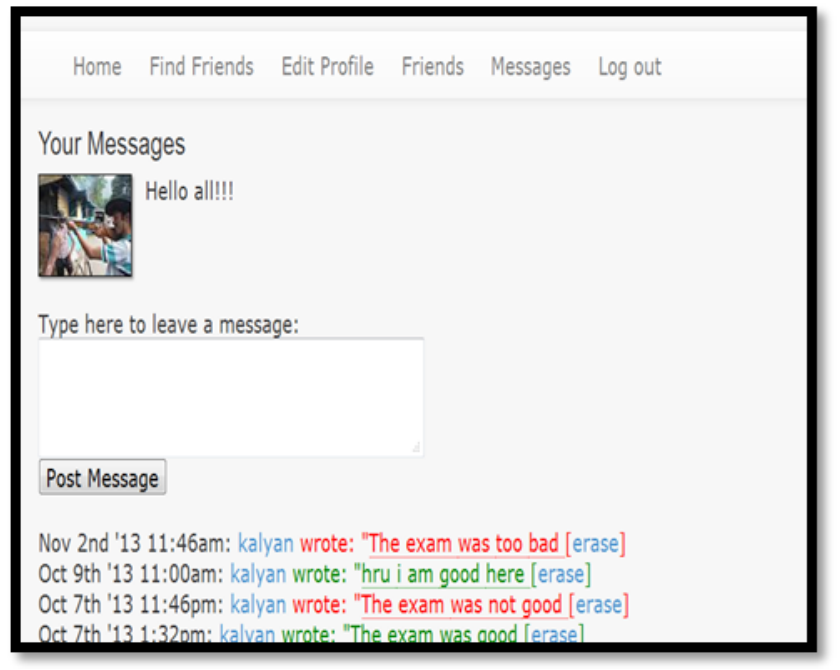

Figure 1. Messages displayed in colour depending on their polarity with that of the words in the column name "short_forms" in the database. If a match is found, the corresponding word in the "full_forms" column is retrieved and is stored in a HTML "acronym" tag. Each word in the message is enveloped with an acronym tag. If any match is found for that word in the MYSQL table, the corresponding full form is stored in the acronym tag. Otherwise, the word itself is stored in the acronym tag. Ex: <acronym title="how are you" $>$ hru $</$ acronym $>$. The above example will be the HTML plain text returned by the PHP page after processing the word "hru" which means "how are you".

The following is the algorithm for Tooltip module,

function extract_acro(String s)

begin

Var temp

temp=mysql_query(select $*$ from table where short_ form $=$ 's')

if(number of rows returned to temp=1)

then

return the long form

if (number of rows returned to tem $p=0$ )

then

end

return the string as it is

Figure 2 shows the working of tooltip translator. If the pointer is placed on a word, its original form is displayed in the tooltip as shown in the figure.

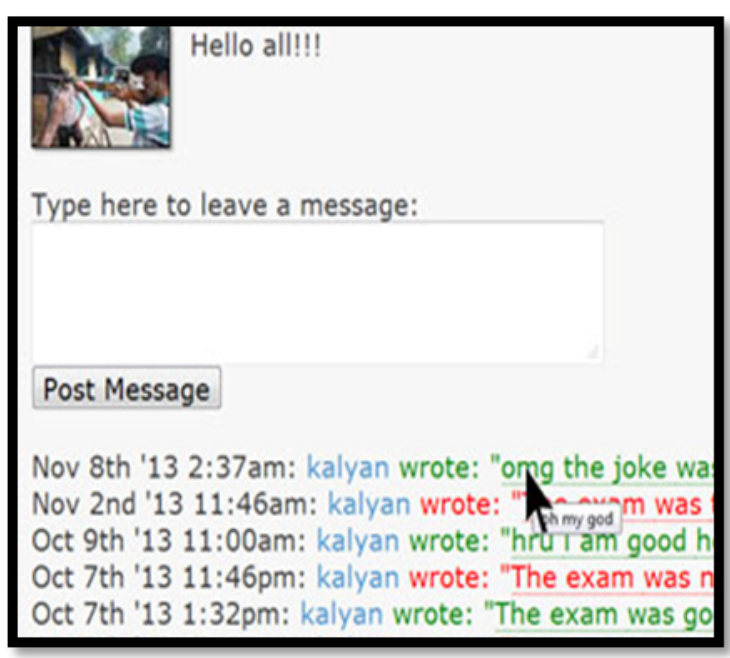

Figure 2. Tooltip showing the full form of a compressed word 


\subsection{Trends Module}

People can determine current trend with respect to any aspect or a person by simply using the trends module. We create two variables with names "p" and " $n$ ". Trends module inputs a word or a set of words and searches all the messages from all the users. It finally returns the overall summarized polarity of the subject that has been entered in the text box.

For example: If we type "Sachin Tendulkar" in the search box in trends page, the page returns a message that shows whether the trend "Sachin Tendulkar" has a positive opinion in public or not. If the trend has a positive opinion, what is positive polarity percentage? In this module, the entered keyword is matched with all the messages from all the users in the site. If a match is found, the polarity of that message is determined. If the polarity is positive, the positive flag is incremented by 1 . If the message is negative, the negative flag is implemented by 1 . Finally the overall polarity of the trend is determined by,

$$
\text { trend_polarity }=[p /(p+n)] * 100
$$

The overall trend is determined as positive, negative or neutral depending on whether the value of trend_polarity is $>50,<50$ or $=50$ respectively.

The following is the algorithm for Trends module,

$$
\begin{aligned}
& \text { function trends(String trend) } \\
& \text { begin } \\
& \text { var temp } \\
& \text { var pos }=0, n e g=0 \\
& \text { temp=mysql_query(select } * \text { from messages) } \\
& \text { while not end of messages table } \\
& \text { var messages[]=fetch messages with string trend } \\
& \text { in them trend_meter[]=sentiment(messages[]) } \\
& \text { for } i=0 \text { unti } i<\text { messages.length } \\
& \text { begin }
\end{aligned}
$$$$
\text { if trend_meter }[i]>50
$$

then

$$
\text { pos }++
$$$$
\text { if trend_meter }[i]<50
$$

then

end

$$
\text { neg++ }
$$

var trend_polarity

trend_polarity $=[$ pos $/($ pos $+n e g)] * 100$

return trend_polarity

end
Home Find Friends Edit Profile Friends Trends Messages Log out find trends

The trend tendulkar has positive response with $66.666666666667 \%$ positiveness.

Figure 3. Trends module

\section{Conclusion and Future Work}

In this paper, we have implemented sentiment analysis using PHP and MYSQL. Hence, complex tasks like opinion mining can be performed in a simpler way using the power of PHP programming language. Public opinion about any subject can be determined using the trends module. In future, an effective PHP toolkit can be intended for natural language processing that can perform all tasks like sentence tokenization, sentence splitting, Parts-ofSpeech tagging etc.

\section{References}

1. NamrataGodbole, Manja Srinivasaiah and Steven Skiena "Large-Scale Sentiment Analysis for News and Blogs", International conference on Weblogs and social media, March 26-28, 2007.

2. Bing Liu, "OPINION MINING", 2007.

3. Viktor Hangya, G'abor Berend, Rich'ard Farkas SZTE"NLP: Sentiment Detection on Twitter Messages", Proceedings of the Second Joint Conference on Lexical and Computational Semantics $(*$ SEM), Volume 2 : Seventh International Workshop on Semantic Evaluation (SemEval 2013), pp: 549-553.

4. Alan Ritter, Sam Clark, Mausam and Oren Etzioni, "Named Entity Recognition in Tweets: An Experimental Study", Conference on Empirical Methods in Natural Language Processing (EMNLP), Edinburgh, UK, July 2011.

5. Apoorv Agarwal, BoyiXie, Ilia Vovsha, Owen Rambow, Rebecca Passonneau, "Sentiment Analysis of Twitter Data", In the proceedings of Workshop on Language in Social Media, ACL, 2011.

6. Jinan Fiaidhi, Osama Mohammed, Sabah Mohammed," Mining twitterspace for information: Classifying sentiments programmatically using Java”, ICDIM 2012:303-308.

7. Jinan Fiaidhi, Osama Mohammed, Sabah Mohammed, "Opinion Mining over Twitterspace: Classifying Tweets Programmatically using the R Approach", ICDIM 2012:313-319

8. Xujuan Zhou, Xiaohui Tao, Jianming Yong, Zhenyu Yang," Sentiment Analysis on Tweets for Social Events", Computer 
Supported Cooperative Work in Design (CSCWD), IEEE 17th International Conference on, 27-29 June 2013, pp: $557-562$

9. Guruprakash. J, Krithika L.B, “TOOLTIP TRANSLATOR FOR ELEARNING COURSEWARE TO IMPROVE LEARNER'S VOCABULARY", 4th International Conference on Education and New Learning Technologies, 2-4 July, 2012

10. Weijian Ni and Yalou Huang -"Extracting and Organizing Acronyms based on Ranking, Intelligent Control and Automation", WCICA 2008 7th World Congress on, June 2008, pp: 25-27.

11. R. Bowden, P. Halstead, G. Rose - "Dictionaryless English plural noun singularisation using a corpus-based list of irregular forms", in the 17th International Conference on English Language Research on Computerized Corpora, Netherlands, 1996.

12. T. Chang, H. Schutze, B. Altman - "An online dictionary of abbreviation from MEDLINE", Journal of the American Medical Informatics Association, 2002; 9, 612-620.
13. S. Larkey, P. Ogilvie, A. Price, B. Tamilio, Acrophile - "An automated acronym extractor and server", in the 15th ACM Conference on Digital Libraries, 2000.

14. Y. Park, J. Byrd Hybrid - "Text mining for finding abbreviations", Proceedings of EMNLP, 2001.

15. J. Pustejovsky, J. Castano, B. Cochran, M. Kotecki, "Automatic extraction of acronym-meaning pairs from MEDLINE databases", Proceedings of Medinfo, 2001.

16. K. Taghva, J. Gilbreth, "Recognizing acronyms and their Technical Report”, ISRI (Information Science Research Institute) UNLV, 1999.

17. H. Yu, G. Hripcsak, C. Friedman, "Mapping abbreviations to full forms in biomedical articles", Journal of the American Medical Informatics Association, 2002; 9(3), 262-272.

18. J. Xu, Y. Huang, "A machine learning approach to recognizing acronyms and their expansions" - Proceedings of the 4th ICMLC, 2005. 